\title{
Comparison of different treatments used for polycystic ovary syndrome
}

\author{
Miriam C. Llamas ${ }^{1 *}$, Javier Méndez Montejano', Maritza Del C. Mudarra Vergara ${ }^{2}$, \\ Aldhara Z. Cordero Muñoz ${ }^{1}$, María G. Ramírez Vargas ${ }^{1}$, Andrea Lira Otero ${ }^{1}$, \\ Luis A. Pineda Martínez ${ }^{1}$, José M. Zepeda Torres ${ }^{1}$, Miriam C. Pérez Díaz ${ }^{3}$
}

\author{
${ }^{1}$ School of Medicine, Universidad Autónoma de Guadalajara, Guadalajara, Jalisco, México \\ ${ }^{2}$ Medicine Program, Academic Unit Health Sciences, Universidad Autónoma de Guadalajara, Guadalajara, Jalisco, \\ México \\ ${ }^{3}$ School of Medicine, Universidad del Valle de México, Zapopan, Jalisco, México
}

Received: 31 December 2021

Revised: 14 January 2022

Accepted: 15 January 2022

\section{*Correspondence:}

Miriam C. Llamas,

E-mail: miriam.llamas@edu.uag.mx

Copyright: (C) the author(s), publisher and licensee Medip Academy. This is an open-access article distributed under the terms of the Creative Commons Attribution Non-Commercial License, which permits unrestricted non-commercial use, distribution, and reproduction in any medium, provided the original work is properly cited.

\begin{abstract}
Polycystic ovary syndrome (PCOS) is a disease that currently affects many women of reproductive age. In recent years, the incidence of PCOS has increased, affecting $4 \%$ of all women worldwide, with a prevalence of $6 \%$ in Mexico. PCOS is a condition characterized by different metabolic, reproductive and hormonal disorders such as hyperandrogenism, chronic anovulation, menorrhagia or infertility. Patients commonly develop clinical alterations such as hirsutism, acne and in some cases, they become overweight or obese. Different medications and therapeutic methods from different literatures were evaluated, both pharmacological such as inositol, metformin, resveratrol, simvastatin, dapagliflozin, which showed great improvement, decreasing the levels of hyperandrogenism in patients, as well as nonpharmacological, of which significant improvements were found with a change in lifestyle, such as exercise, ketogenic diet and herbal medications such as chamomile and cinnamon, which showed a positive change in patients. It is important to make a diffusion and early diagnosis of PCOS, since in this way it will be possible to have a timely treatment, which can be individualized according to the characteristics and needs of each patient.
\end{abstract}

Keywords: Polycystic ovary syndrome, Treatment, Comparison, Hyperandrogenism

\section{INTRODUCTION}

PCOS is a set of symptoms and signs caused by a common metabolic and reproductive disorder in women. It is estimated to occur in $4 \%$ of women of childbearing age worldwide, however, other reports show an estimate of $10 \%$ in populations at higher risk. In Mexico, the prevalence of PCOS is $6 \%$, with higher frequency in women with obesity and diabetes, both related to insulin resistance. ${ }^{1}$ Current findings corroborate the concept that insulin resistance reflects ectopic lipid accumulation, particularly in the liver, and precedes the development of disorders such as type 2 diabetes and non-alcoholic fatty liver disease. Increased liver fat and insulin resistance are common findings in both obese and non-obese adolescents with PCOS. ${ }^{2}$

PCOS is considered a clinically important condition characterized by menstrual disorders such as chronic anovulation, menorrhagia, with the consequent infertility. The ovaries are polycystic and there is hyperandrogenism in patients, which is associated with obesity as well as insulin problems. ${ }^{3}$ There are many comorbidities involved, so it leads to a multidisciplinary management and treatment, most of them are treatable and early diagnosis of patients improves their reproductive prognosis. 
Currently, pharmacological and non-pharmacological treatments for PCOS have aimed to achieve ovulation, normalize menstrual cycles and eliminate hirsutism and acne as far as possible, obtain an acceptable weight loss, treat dyslipidemia and hyperglycemia to reduce the risk of cardiovascular disease. The treatment will depend on the reason for the patient's consultation. It has been proven that changes in eating habits and increased exercise, supported by a multidisciplinary team, including psychologists and nutritionists, help these patients in a positive way. ${ }^{4}$ The loss of $10 \%$ of body weight improves menstrual function and fertility, in parallel to the improvement of insulin resistance and metabolic alterations. Women with PCOS are known to have a low basal metabolism compared to healthy women and patients commonly have difficulty with weight loss even with low calorie diets. The aim of a change in lifestyle was to prevent the development of diabetes in patients with this pathology, which had been shown that improving glucose levels causes a decrease in hyperinsulinemia, causing a decrease in androgen levels. ${ }^{5,6}$ In addition, excess androgens contributed to altered negative feedback of ovarian hormones on luteinizing hormone (LH) pulse rate and thus fuelled an arrhythmic reproductive cycle. ${ }^{7}$

\section{Justification and approach}

This pathology represented a real challenge worldwide, since its presentation figures were high, mainly in women of reproductive age, of which no predominance between races or nationalities was observed. ${ }^{3}$ The importance of its diagnosis and treatment relied in the possible complications it entailed such as insulin resistance, infertility, diabetes mellitus (DM), obesity, cardiovascular disease and even endometrial cancer. ${ }^{8,9}$

The main objective of this research was to make a description of the different treatments that currently existed for women diagnosed with PCOS, in this way it will be possible to find the most appropriate treatment scheme, with greater effectiveness for the patients, to reach the purpose of helping patients to improve their quality of life.

\section{Theoretical framework}

PCOS is an endocrine and metabolic disorder. There are four recognized phenotypes of PCOS: (1) hyperandrogenism+oligo-anovulation+polycystic ovarian morphology, (2) hyperandrogenism+oligo-anovulation, (3) hyperandrogenism+polycystic ovarian morphology, and (4) oligo-anovulation+polycystic ovarian morphology. The treatment in PCOS depends on the patients' phenotypes, concerns and goals and should focus on improving fertility; suppressing and counteracting androgen secretion and action; and improving metabolic status. The most frequent problem of these patients was infertility, highlighting that oligo-anovulation may be present in $70 \%$ of cases. Specific mechanisms related to genetic and epigenetic bases, neuroendocrine dysfunction, hyperandrogenism, metabolic alterations, follicular development, follicular arrest, oocyte competence and quality, recurrent miscarriages, endocrine disruptors (environmental factors) as well as chronic inflammation stand out. ${ }^{10,11}$ Clinical symptoms, in addition to comprising reproductive disorders included metabolic disorders such as dyslipidaemia, hypertension, insulin resistance, compensatory hyperinsulinemia, type $2 \mathrm{DM}$, gestational diabetes as well as increased risk for cardiovascular disturbances and mental signs. ${ }^{12}$

According to the last-mentioned point, current evidence mentioned that there was a chronic degree of low-grade inflammation in patients with this pathology, therefore, it was considered as a proinflammatory condition. Among the main proinflammatory factors found, C-reactive protein (CRP), interleukin 6 (IL-6), tumor necrosis factor $\alpha(\mathrm{TNF}-\alpha)$ and interleukin 18 (IL-18) were described. ${ }^{13}$

Similarly, it had been described that dysbiosis, that was, the imbalance of the intestinal microbiota, played an important role in the development of this syndrome since the activity of sexual hormonal regulation influenced directly the number and diversity of the microbiota, the evidence showed significant differences in composition, configuration and diversity of the intestinal microbiota in healthy women compared to women suffering from PCOS. ${ }^{14}$ All these pathophysiological mechanisms involved were interrelated, interfering in such a way that they became a vicious circle, where each one of them, especially the metabolic alterations, can negatively affect the fertility and reproductive outcome of the woman.

Different pharmaceutical treatments have been proposed for PCOS. However, they present disadvantages such as adverse effects, low patient compliance with long-term pharmaceutical treatments, low efficacy and contraindications in some cases; therefore, complementary treatments may be suitable alternatives. Nowadays, oral contraceptives (OCPs) are the most common options for the treatment of PCOS, which reduce free androgens in the blood and suppress gonadotropin secretion. ${ }^{15}$

Among the treatments described for this pathology, the drugs that were considered as the first choice, along with changes in lifestyle, were inositol or resveratrol as well as metformin and simvastatin. Inositol is part of a group of nutritional supplements, within the pathophysiology of PCOS, there was an alteration either in the availability or metabolism of inositol, this contributed to the presentation of insulin resistance. ${ }^{9}$

In one study, it was observed that by providing this drug, women affected with the pathology were able to improve their ovulatory functions, specifically seeing an increase in ovulation rate. This drug was tested in two different presentations: Myo-inositol and D-chiro-inositol, however, the response rates did not vary and the benefits were the same. Hormonally, inositol achieved a decrease in total androgen levels, total testosterone, free 
testosterone, dehydroepiandrosterone, decreased fasting serum insulin levels, fasting glucose and homeostatic model assessment for insulin resistance (HOMA). ${ }^{9}$

PCOS represented a state of chronic low-grade inflammation, therefore, it was essential to control the pathology taking into consideration this action in the body. Increased levels of CRP, IL- 6, TNF- $\alpha$ and IL- 8 have been observed. Based on pharmacological measures, the use of resveratrol was observed in a group of PCOS patients, this drug was a natural polyphenol found in grapes, nuts and berries, which had the ability to inhibit proinflammatory genes that produced interleukin $1 \beta$ (IL-1 $\beta$ ), IL-6 and cyclooxygenase-2 (COX-2), in addition to having antiinflammatory, anti-apoptotic and a P53 anti-tumor suppressor function. ${ }^{13}$

While evaluating the efficacy of resveratrol, participants had their levels of IL- 6 , IL- $1 \beta$, TNF- $\alpha$, IL-18, nuclear factor $\kappa$-light-chain-enhancer of activated B cells $(\mathrm{NF}-\kappa \mathrm{B})$ and CRP quantified. The treatment lasted for 40 days, at the end of this, a new quantification of these levels was done, finding a statistically significant decrease of TNF- $\alpha$, IL-18, NF- $\kappa$ B and CRP, this due to the blockade of IL- $1 \beta$, TNF- $\alpha$ and an inactivation of NF- $\kappa B$. This could be a great pharmacological measure on its own, however, benefits were only seen with the aspects described above, plus its action varied depending on tissue type, intensity of inflammation and quantification of cytokines. ${ }^{13,16}$

Talking about metformin, it performed its hypoglycemic action mainly at the level of the liver, small intestine, skeletal muscle, adipose tissue and ovary. One of the best known effects of metformin was that it acted at the mitochondrial level in the liver, suppressing adenosine triphosphate (ATP) production and consequently reducing gluconeogenesis. ${ }^{4,17}$ Metformin administered together with simvastatin improved insulin resistance, fasting insulin, insulin sensitivity index, hyperandrogenaemia and hirsutism, even a significant decrease in androgen levels was described in patients with PCOS compared to single administration of metformin, similarly it had been described that it produced improvements in lipid profiles and endothelial function thereby reducing cardiovascular morbidity and mortality in patients with PCOS. ${ }^{18,19}$

Regarding the different affectations involved in PCOS, ovulation and fertile alterations were included. This syndrome was responsible for approximately $80-90 \%$ of existing anovulatory infertility. ${ }^{20}$ Although the mechanism that causes anovulation in PCOS is still unknown, it is considered that, for menstrual irregularities, pulsatile alterations of gonadotropin-releasing hormone (GnRH) and inadequate secretion of gonadotropins were mainly involved. ${ }^{3}$ Regarding the information above described, Rezk based his study on the use of clomiphene citrate (CC) combined with metformin compared to the use of letrozole, to assess ovulation induction in PCOS patients. ${ }^{20}$
Anovulation was likely to be the result of insulin resistance, because a substantial number of patients with PCOS anovulation resumed ovulatory cycles when treated with metformin, an insulin sensitizer. ${ }^{3}$ Androgen excess can be biochemical or clinically manifested. Biochemical hyperandrogenemia was present in $60-80 \%$ of adults with PCOS, attributed primarily to excess androgen production by the ovaries, with the adrenal glands and peripheral adipose tissue contributing to a small degree. Androgen excess can be clinically manifested as hirsutism. ${ }^{21}$ According to a randomized placebo clinical trial, it had been shown that androgen and hirsutism score had an improvement in overweight women with PCOS after vitamin $\mathrm{D}_{3}$ treatment. ${ }^{22}$ It was known that in patients who desired pregnancy but remained anovulatory, letrozole, $\mathrm{CC}$ or some other ovulation-inducing drug should be given as a first choice and metformin in conjunction with $\mathrm{CC}$ as a second line indication in patients with clinical manifestations of PCOS, resistance to CC and anovulatory infertility. ${ }^{3,23}$ In the Rezk study, letrozole was associated with higher ovulation and clinical pregnancy rates compared with the combination of $\mathrm{CC}$ and metformin in CC-resistant PCOS. Therefore, letrozole emerged as a better and more acceptable ovulation-inducing treatment in these patients. In addition, there was a higher rate of multiple pregnancies. ${ }^{20}$ So, at present, letrozole, which was an aromatase inhibitor, was now considered the first line for ovarian induction in PCOS patients.

On the other hand, a meta-analysis conducted by Creanga studied the efficacy of metformin, used both alone and in combination with $\mathrm{CC}$, with respect to ovulation, early pregnancy achievement and live births. The meta-analysis concluded that metformin alone improved the odds of ovulation in women with PCOS, but was not helpful in achieving clinical pregnancy. Therefore, the findings suggested that combination therapy, using metformin and $\mathrm{CC}$, increased the likelihood of ovulation and early pregnancy compared with $\mathrm{CC}$ alone, especially among patients with obesity and CC resistance. Even so, it should be noted that this combined therapy may not improve the odds of live births, bearing in mind that certain patients with PCOS who suffered from infertility will have as a target of interest the probability of having live births. ${ }^{24}$

In a study by Løvvik the use of metformin in pregnant patients with PCOS was examined and the study was designed to explore the possible beneficial effects of metformin on preeclampsia, preterm delivery and gestational diabetes. In this study, it was observed that pregnant patients treated with metformin had more diarrhea than those in the placebo group at 19 and 28 weeks of gestation, but later in pregnancy there was no difference in this regard. ${ }^{25}$ Rezk also studied that the group of patients treated with metformin and $\mathrm{CC}$ experienced adverse effects, mainly from headache in $27.45 \%$ of patients, gastric discomfort in $45.09 \%$ and breast tenderness in $17.65 \% .{ }^{20}$ Likewise, Løvvik showed that metformin had no effect on the incidence of gestational diabetes or on the need for insulin treatment, although the 
results suggested that it may reduce the risk of late miscarriage and preterm delivery in pregnant patients with PCOS, as well as being an inexpensive, tolerable and widely available treatment. ${ }^{25}$

Ovarian drilling by laparoscopy, also known as ovarian electro-puncture, which consisted of making multiple perforations on the surface of the ovary, in order to reduce its volume and stimulate the selection of an ovulatory follicle for the release of an egg, was a treatment that can also be considered as a second line treatment, with similar results to those obtained in 3-6 cycles of ovarian induction. ${ }^{26}$ Ovarian drilling provided benefits related to a lower risk of ovarian hyperstimulation syndrome (OHSS). Some studies suggested that its effect could last over time, allowing to obtain a spontaneous pregnancy for women with PCOS and even to obtain more than one pregnancy.

As a highlighted note, in 2019, the Instituto Mexicano del Seguro Social (IMSS), in the maternal-fetal service of the hospital of obstetrics and gynecology number 3 of the Centro Médico Nacional (CMN) La Raza, performed this technique for the first time with excellent results. ${ }^{27}$

It was known that treatment goals for PCOS can be listed to correct hyperandrogenism, menstrual dysfunction and infertility. In addition, OCPs, long-acting GnRH analogs and agents that improved insulin sensitivity, can be used in androgen-lowering therapy. ${ }^{28}$ Recently used combination OCPs such as ethinylestradiol+cyproterone acetate (EE+CA), EE+desogestrel and EE+drospirenone have shown promising results in reducing androgen levels and regulating menstruation. ${ }^{6}$ From these, cyproterone acetate stood out, which can block peripheral androgen receptors in target organs, reduced ovarian androgen production and decreased plasma levels of free testosterone. ${ }^{29}$ This drug was especially used for patients who have acne, hirsutism and hyperandrogenism. ${ }^{30}$

A study by Abdel-Maboud established a competition between antiandrogen drugs, OCPs, antidiabetics, cholesterol modulators and combinations between categories. The study observed that flutamide, an antiandrogen, demonstrated efficacy in improving anthropometric, androgenic and lipid parameters. It was also studied that EE+CA, an antiandrogen with an OCP, showed the greatest efficacy in improving androgenic parameters; however, it did not show superiority in the other parameters. The analysis revealed that most combinations were not promising, for example, combinations of flutamide+metformin, $\mathrm{EE}+$ flutamide+levonorgestrel, $\mathrm{EE}+\mathrm{CA}+$ metformin and $\mathrm{EE}+\mathrm{CA}+$ orlistat were inferior to either agent alone. Still, it remained questionable whether a future combination of flutamide+EE+CA may create a better potential, so further studies were required. With the investigation, a superiority of flutamide in improving metabolic and hormonal parameters and a higher efficacy of EE+CA only in improving hormonal parameters was demonstrated. In addition, almost all the interventions were comparable in terms of improvements in female hormone levels, fasting serum glucose, HDL, glucose and insulin. ${ }^{6}$ Metformin, $\mathrm{EE}+\mathrm{CA}$ and metformin+EE+CA were subjected to analysis in Ünal's study, to determine whether carotid intima-media thickness (IMT), HOMA, apelin, adiponectin and CRP levels in PCOS cases differed from the control group and to evaluate how the above parameters responded to the 3 treatments mentioned in his study. With the analysis established, it was detected an increase in carotid IMT in PCOS cases, which was an early symptom of atherosclerosis; low serum levels of adiponectin and apelin; high CRP levels; no significant decrease in carotid IMT in the 3 post-treatment groups; an increase in serum apelin levels in all 3 treatment groups; and although, adiponectin level increased in the metformin group and in the metformin+EE+CA group, it did not change in the EE+CA group. However, further studies were needed to evaluate clinical and subclinical atherosclerosis in PCOS cases. ${ }^{28}$ George in his study, compared the efficacy of metformin and EE+CA in the treatment of hyperandrogenism in PCOS patients, in which he also analyzed the efficacy of both drugs in relation to parameters such as testosterone, LH, follicle stimulating hormone (FSH), fasting insulin, dehydroepiandrosterone sulfate (DHEAS), anti-Müllerian hormone, body mass index (BMI) and waist:hip ratio (WHR). In the study, it was found that testosterone, LH, FSH and DHEAS parameters were reduced when patients were treated with $\mathrm{EE}+\mathrm{CA}$ than in the case of metformin. In addition, fasting insulin, BMI and WHR were much more significantly reduced with metformin treatment compared to $\mathrm{EE}+\mathrm{CA}$. Both drugs showed overall benefits in clinical complaints of PCOS, however, George's data suggested that EE+CA was a more effective way to treat hyperandrogenism and menstrual dysfunction, considering that improvement in hirsutism, improvement in hyperandrogenemia and restoration of regular menstrual cycles occurred more frequently with $\mathrm{EE}+\mathrm{CA}$ than with metformin. In addition, metformin clearly outperformed $\mathrm{EE}+\mathrm{CA}$ in improving insulin resistance, reducing BMI and also in improving ovulation. With the above, George was able to conclude that $\mathrm{EE}+\mathrm{CA}$ proved to be much more effective in controlling hyperandrogenism compared to metformin. ${ }^{31}$

As it was known, currently used treatments for PCOS focus on treating PCOS-associated metabolic syndrome (MS), anovulation, endometrial dysfunction and hyperandrogenism. ${ }^{23}$ In addition, endocrine and metabolic disturbances such as hyperandrogenism, hyperinsulinemia and dyslipidemia, may be closely involved in PCOS-associated oxidative stress.

Regarding total antioxidant capacity (TAC) in serum as an indicator about the overall protective effect of antioxidants in body fluids, cell membranes and other cellular components against oxidative injury as well as malondialdehyde (MDA), Borzoei demonstrated that cinnamon supplementation had an antioxidant capacity, where the ethanolic extract present in cinnamon decreased lipid peroxidation induced by carbon tetrachloride and 
consequently, there was a decrease in oxidative stress markers. This turned out to be very relevant in the treatment of the disease, since in PCOS, low-density lipoproteins (LDL), triglycerides (TGL) and total cholesterol:high-density lipoproteins (HDL) ratio, may be increased, as well as decreased HDL. ${ }^{1}$

Thus, lipid changes in PCOS increased the risk of cardiovascular disorders. ${ }^{3}$ Borzoei observed that cinnamon supplementation caused a significant increase in TAC levels and decrease in MDA levels compared to the placebo group studied as well as a decrease in serum levels of total and LDL cholesterol and an increase in HDL levels. Due to the information given above, it was proposed that the antihyperlipidemic activity of cinnamon could be due to its high content of polyphenols, which inhibited the intestinal absorption of cholesterol with consequent hypocholesterolemic activity. ${ }^{1}$

In a study conducted by Heidary, lipid parameters such as TGL, cholesterol, HDL, LDL, LH:FSH ratio, total testosterone-testosterone and DHEAS were used in relation to chamomile consumption in PCOS patients. ${ }^{12}$ It was known that insulin as well as LH, stimulated androgen production in ovarian theca cells. Thus, the ovaries secreted increased amounts of testosterone and androstenedione. Specifically, high free testosterone was observed in $70-80 \%$ of women with PCOS and 25-65\% have increased DHEAS. In turn, high androstenedione contributed to increased estrogen levels through biotransformation of androgens to estrogens by aromatase. ${ }^{3}$ In the results of Heidary's study, chamomile consumption decreased serum LDL and TGL and increased HDL in PCOS patients, although this change was not significant compared to the control group. Also, there was a slight increase in DHEAS, which was not significant in either studied group. Heidary was able to demonstrate that chamomile consumption was effective and caused a significant decrease in serum testosterone levels. However, no significant changes were found in lipid parameters, LH:FSH ratio, nor DHEAS level. ${ }^{12}$

A new study, conducted by Ghanei proposed to study the association of OCPs with probiotics, in which it was observed that the combination of both significantly reduces inflammation associated with PCOS by reducing CRP and IL-6 as well as weight in patients with PCOS. The study especially analyzed the effect of cyproterone acetate with probiotic supplements, employing 4 strains of Lactobacillus, using Lactobacillus acidophilus, Lactobacillus plantarum, Lactobacillus fermentum, and Lactobacillus gasseri. The research found immunological and inflammatory changes in relation to this treatment in women with PCOS after 3 months. Lactobacillus supplementation showed beneficial effects on anthropometric indices and cytokine balance in PCOS patients. In addition, interleukin 10 (IL-10) was observed to be significantly elevated in response to probiotics in PCOS with cyproterone acetate treatment, so this elevation may help explain the potential mechanism of inflammation reduction given by probiotics. ${ }^{30}$

Behboudi-Gandevani conducted an investigation of the effect of phlebotomy on clinical and biochemical parameters in women with PCOS compared to OCPs containing cyproterone acetate. As a result, both treatments had similar beneficial effects on insulin resistance as well as on androgen profiles. Still, cyproterone acetate-containing OCPs were found to be more effective in treating menstrual irregularity and phlebotomy had fewer adverse effects. At the end of the study, Behboudi-Gandevani found that phlebotomy therapy was associated with decreased insulin resistance and hyperandrogenic status in women with PCOS, which was achieved with proper vein selection and a careful venipuncture procedure to help minimize likely vasovagal reactions. ${ }^{29}$

Pharmacological treatments were considered as the first line of management, however, when combined with diet and exercise, they helped to achieve a greater benefit, in less time and even a better response to the medications used. A change in diet helped to control the increase of visceral and subcutaneous fat, in addition to improving glucose levels, insulin, HOMA index, total testosterone, FSH and achieving an increase in HDL, estradiol and progesterone. These results were possible thanks to the use of a ketogenic diet, which was a protocol in which carbohydrates were less than $30 \mathrm{~g}$ per day or $5 \%$ of total energy intake, relative to protein and fat intake. ${ }^{32}$

In one study, this diet was modified and called KEMEPHY, which was a eucaloric ketogenic Mediterranean diet with a protocol of 1,600-1,700 $\mathrm{kcal} / \mathrm{day}$, applied for 12 weeks, there were no restrictions in the consumption of green leafy vegetables, pumpkin, cucumber and eggplant; the amount of meat (120 g), egg (2 per day), carbohydrates (3.5 g) and fish (20 g) were limited. In addition to this, 4 food supplements and herbal extracts were consumed on a daily basis. In addition to the described results, an average weight loss of $9.43 \mathrm{~kg}$ was also achieved, whereby an adequate diet is considered as an indispensable cofactor together with pharmacological management. ${ }^{32}$

Continuing with lifestyle modifications, exercise had been shown to be part of the recommendations as a first-line treatment for women with $\mathrm{PCOS} .^{8}$ A recent one-year study showed that weight loss, defined by a reduction in BMI $>0.2$, was associated with decreased testosterone levels, increased sex hormone binding globulin (SHBG) and reduced amenorrhea from $69 \%$ to $27 \%$. Likewise, the prevalence of MS decreased from $35 \%$ to $4 \%$, with reductions in HOMA index, TGL and blood pressure (BP). ${ }^{33}$ Another article compared the benefits that could be found between aerobic exercise, aerobic exercise with acupuncture, aerobic exercise with resistance and high intensity aerobic exercise. These were practiced 3 times a week, with an average duration of 25-60 minutes, for a 
total duration of 12 weeks. At the end of this period of time, no real difference was found between the different modalities of aerobic exercise, however, an improvement in their hormonal and lipid profile was found; this physical activity managed to stimulate the reception of oxygen, the oxidation of free fatty acids, decreased the BMI, improved body composition along with the circulation of glucose as an energy source, in this way, there was an increase in aerobic metabolism. ${ }^{8}$

Many PCOS patients suffered from hirsutism and other dermatological manifestations of hyperandrogenism, so cosmetic or topical treatments should be considered and encouraged. ${ }^{34}$ For hirsutism caused by PCOS, lifestyle changes have shown medium improvement, which had been seen in $95 \%$ of patients, although the benefits have also been seen to be more towards improving overall health. In women with this problem or even acne, OCPs have been shown to be the first-line treatment, only when pregnancy was not being sought; the effects should be seen over a 6-month period of time. Another drug that had shown great improvement rates on hirsutism is spironolactone, which was an aldosterone receptor antagonist. This drug was contraindicated in pregnancy, as it blocked the androgenic action in the formation of the male external genitalia, in such a way that it was necessary to be combined with contraceptives or to encourage abstinence. Similarly, finasteride, which was a $5 \alpha-$ reductase inhibitor, showed a similar effect in doses of 2.5 or $5 \mathrm{mg}$ and a slight improvement at $7.5 \mathrm{mg}$; it was necessary to mention the side effects to patients such as decreased libido, depression or anesthesia and orthostatic hypotension. Similarly, there were topical alternatives for hirsutism such as shaving, depilation, hair bleaching, creams, photoepilation or electrolysis. ${ }^{35}$ Just as hirsutism required suppression of androgen secretion and action as mentioned above, hair bleaching can be used if hirsutism was mild; shaving and chemical hair removal, if moderate; or shaving, if severe. ${ }^{34}$ In addition, if a local effect was sought with medication, topical antiandrogens can be chosen. ${ }^{35}$ Also, if there were patients suffering from acne and alopecia as a result of PCOS, hair transplant surgery may be necessary. However, long-term treatment of acne and alopecia should be carried out with the support of a dermatology specialist. ${ }^{34}$

\section{DISCUSSION}

In this research the elements involved in PCOS were taken into account regarding ovulatory and fertile alterations, the consequent problems of preeclampsia, risks of spontaneous miscarriage, late and/or premature delivery, gestational diabetes as well as factors related to MS, hyperandrogenism, hyperinsulinemia and dyslipidemia, which may be highly linked to the oxidative stress associated with PCOS.

In regards to this, in a study conducted by Rezk a comparison was made where he showed that treatment with letrozole obtained high rates of ovulation induction and pregnancy compared to the combination of CC and metformin. It showed that pregnant patients treated with metformin alone experienced adverse effects such as diarrhea, headache in $27.45 \%$, gastric discomfort in $45.09 \%$ and breast tenderness in $17.65 \%$ and no effect on the incidence of gestational diabetes was found. ${ }^{20}$ Likewise, another study showed that the use of metformin had a reduction in the risk of miscarriage, late miscarriage and preterm delivery in pregnant patients with PCOS. ${ }^{25}$ Herbal medicines not only improved reproductive dysfunctions, but also played a remarkable role in balancing hormonal status and menstrual cycles. The use of Cinnamomum cassia supplement for at least 6 months could normalize menstrual cycles. Cinnamon, as a bioactive medication, produced a reduction in antiMüllerian hormone and had fewer side effects compared to metformin. ${ }^{15}$ Also, metformin alone proved to be better for ovulation in women with PCOS, but it was not helpful for the achievement of clinical pregnancy. Creanga's findings showed that combination therapy, using metformin and $\mathrm{CC}$, increased ovulation and early pregnancy rates compared with $\mathrm{CC}$ alone, especially in PCOS patients that suffered from obesity and CC resistance and that this combined therapy may not be better to obtain live births. ${ }^{24}$

Following the observations in pharmacological treatments, Pundir et al proved that inositol achieved a decrease in different hormonal components such as total androgens, different types of testosterone, serum levels of insulin, glucose and HOMA. These results were similar to those obtained by Paoli et al however, they only relied on the use of a different diet to achieve such changes. ${ }^{9,32}$ In the case of resveratrol, it only showed consistent improvements in the pathophysiological explanation of PCOS from a state of chronic inflammation, without presenting changes at a significant clinical level, in addition to presenting more disadvantages such as being tissue-dependent, the intensity of inflammation and cytokine levels. ${ }^{13}$

Abdel-Maboud's investigation presented a superiority of flutamide in improving metabolic and hormonal parameters and a higher efficacy of EE+CA only in improving hormonal parameters. ${ }^{6}$ A group of patients with PCOS treated with metformin, showed significant improvements in parameters as IMT, HOMA, apelin, adiponectin and CRP levels, in comparison with patients treated with metformin+EE+CA or with $\mathrm{EE}+\mathrm{CA} .{ }^{28}$ On the other hand, George demonstrated that $\mathrm{EE}+\mathrm{CA}$ proved to be more effective in the control of hyperandrogenism compared to metformin. ${ }^{31}$

On the other hand, it was observed that there are more alternatives to complement the pharmacological treatments that according to the exposed results suggested that they were effective in attenuating the antioxidant, hormonal and metabolic imbalances of PCOS as well as the standard therapeutic strategies. ${ }^{16}$ Borzoei proposed in his study that the antihyperlipidemic activity of cinnamon may be due to its high polyphenol content, inhibiting the 
intestinal absorption of cholesterol, with the subsequent hypocholesterolemic activity, because he observed an increase in TAC and HDL levels and a decrease in total cholesterol and LDL levels. ${ }^{1}$ On the other hand, a study by Heidary also showed similar results to Borzoei's, as he observed that chamomile consumption increased HDL levels and decreased serum LDL and TGL levels, in addition to demonstrating that its use decreased serum testosterone levels in PCOS patients. ${ }^{12}$
A treatment with OCPs with Lactobacillus supplementation for a period of time of 3 months showed benefits on anthropometry and cytokine balance in PCOS patients. ${ }^{30}$ In addition, the study conducted by BehboudiGandevani presented that cyproterone acetate-containing OCPs were found to be more effective in treating menstrual irregularities, while phlebotomy therapy was associated with decreased insulin resistance, hyperandrogenic status and fewer adverse effects in women with PCOS. ${ }^{29}$

Table 1: Advantages and disadvantages of the different treatments used in PCOS.

\begin{tabular}{|c|c|c|}
\hline Treatment & Advantages & Disadvantages \\
\hline Inositol & $\begin{array}{l}\text { Decrease in total androgen, free testosterone, } \\
\text { total testosterone levels, and } \\
\text { dehydroepiandrosterone; reduction in fasting } \\
\text { serum insulin, fasting glucose, and HOMA } \\
\text { levels }\end{array}$ & $\begin{array}{l}\text { No evidence of improvement in conception } \\
\text { or decrease in miscarriages }\end{array}$ \\
\hline $\begin{array}{l}\text { Resveratrol } \\
\text { (polyphenols) }\end{array}$ & $\begin{array}{l}\text { Decrease in TNF- } \alpha, \text { IL- } 18, N F-\kappa B \text {, and CPR } \\
\text { levels; positive effects by the increase in the } \\
\text { number of oocytes, inhibiting apoptosis and } \\
\text { decreasing the follicular atresia }\end{array}$ & $\begin{array}{l}\text { The action varies according to the tissue, } \\
\text { the intensity of the inflammation and how } \\
\text { the cytokines are found }\end{array}$ \\
\hline Metformin & $\begin{array}{l}\text { Significant decrease in body weight; decrease } \\
\text { in fasting plasma glucose; decrease in } \\
\text { testosterone levels and total plasma } \\
\text { cholesterol; improvement in endothelial } \\
\text { function; protective effect against } \\
\text { inflammation; causes vascular relaxation; } \\
\text { reduction of the risk of late miscarriage and } \\
\text { preterm birth; improvement in ovulation in } \\
\text { women with PCOS }\end{array}$ & $\begin{array}{l}\text { In women with obesity, the response to the } \\
\text { treatment is slower; it is not clear if in the } \\
\text { long term, the endothelial improvement it } \\
\text { offers may reduce the incidence of } \\
\text { cardiovascular events in addition to } \\
\text { maintaining this endothelial improvement } \\
\text { over the long term; no improvement in } \\
\text { gestational diabetes; in pregnant women: } \\
\text { hyperemesis, colitis, rectal bleeding, } \\
\text { tachycardia, dental problems; in offspring: } \\
\text { fetal malformations, perinatal fetal death, } \\
\text { jaundice, hypoglycemia, respiratory } \\
\text { problems, skeletal abnormalities }\end{array}$ \\
\hline $\begin{array}{l}\text { Simvastatin } \\
\text { (statins)+metformin }\end{array}$ & $\begin{array}{l}\text { Improvement in lipid and inflammation } \\
\text { parameters; significant reduction of total } \\
\text { testosterone; reduction in LDL cholesterol } \\
\text { levels }\end{array}$ & $\begin{array}{l}\text { Did not improve insulin sensitivity or } \\
\text { reduce levels of hyperandrogenism; no } \\
\text { obvious effect on fasting glucose levels }\end{array}$ \\
\hline $\begin{array}{l}\text { Clomiphene } \\
\text { citrate+metformin }\end{array}$ & $\begin{array}{l}\text { Ovulation induction (with low rates); early } \\
\text { pregnancy }\end{array}$ & $\begin{array}{l}\text { Headache; gastric discomfort; breast } \\
\text { tenderness; may not improve the odds of } \\
\text { live births }\end{array}$ \\
\hline Saxagliptin & $\begin{array}{l}\text { Decreases glycosylated hemoglobin levels in } \\
\text { PCOS patients with DM; this medicine, in the } \\
\text { company of the family of medicines from the } \\
\text { hormone glucagon-like peptide-1 (GLP-1) } \\
\text { receptor agonists and dipeptidyl peptidase- } 4 \\
\text { (DPP4) inhibitors, causes the same effect in } \\
\text { patients }\end{array}$ & $\begin{array}{l}\text { Increases insulin levels by mimicking the } \\
\text { action of incretins }\end{array}$ \\
\hline $\begin{array}{l}\text { Saxagliptin+metformi } \\
\text { n }\end{array}$ & $\begin{array}{l}\text { Normalizes impaired fasting glucose and oral } \\
\text { glucose tolerance test (OGTT) }\end{array}$ & $\begin{array}{l}\text { Causes nausea and vomiting in certain } \\
\text { patients with poor tolerance to the drug }\end{array}$ \\
\hline Dapagliflozin & $\begin{array}{l}\text { Reduction in the renal absorption of glucose } \\
\text { (lowering the threshold) and increase in its } \\
\text { urinary excretion; maintains normal glucose } \\
\text { levels by reducing hyperglycemia and } \\
\text { hyperinsulinemia in patients with PCOS }\end{array}$ & $\begin{array}{l}\text { High price; nausea, dizziness; in certain } \\
\text { patients, there have been cases of } \\
\text { respiratory distress }\end{array}$ \\
\hline $\begin{array}{l}\text { Combined oral } \\
\text { contraceptives }\end{array}$ & $\begin{array}{l}\text { Reduction in androgen levels; menstrual } \\
\text { regulation }\end{array}$ & $\begin{array}{l}\text { Adverse effects on lipid metabolism and } \\
\text { carbohydrate intolerance; prothrombotic }\end{array}$ \\
\hline
\end{tabular}


Improvement in hormonal parameters; effective in the control of hyperandrogenism and menstrual dysfunction oterone acetate

Lactobacillus+cyprote rone acetate

rone acetate

Flutamide

Spironolactone

Aldosterone receptor antagonist; adequate control of hirsutism

Beneficial effects on anthropometric indices and cytokine balance; potential mechanism of inflammation reduction

Improvement in metabolic and hormonal parameters

\begin{tabular}{|ll|}
\hline Finasteride & control of hirsutism \\
\hline $\begin{array}{l}\text { Quercetin } \\
\text { (polyphenols) }\end{array}$ & $\begin{array}{l}\text { Functions as a regulator of the cellular } \\
\text { steroidogenic activity, correcting the hormonal } \\
\text { indices; improvement in the antioxidant } \\
\text { capacity of the ovary; reverses PCOS-induced } \\
\text { alterations in lipid profile }\end{array}$ \\
\hline
\end{tabular}

$\begin{array}{ll}\text { Letrozole } & \begin{array}{l}\text { Increase in ovulation induction; higher } \\ \text { pregnancy rates }\end{array}\end{array}$
pregnancy rates

Significant declination in hirsutism scores; improvement in total testosterone levels; significant increase in SHBG level of overweight women with PCOS; significant decrease in free androgen index (FAI); highly significant improvements in serum $25(\mathrm{OH}) \mathrm{D}$

Vitamin $D_{3}$ concentrations; decrease in parathyroid hormone (PTH) level; up-regulation of calcium $(\mathrm{Ca})$ levels; improvement in ovarian ultrasonography; regulation of menstrual cycle; improvement in fertility and reproductive life in overweight women with PCOS

Cinnamon (polyphenols) Protective effect on the oxidant/antioxidant balance; increase in levels of TAC and HDL; decrease in total cholesterol and LDL levels Increase in lipid oxidation; increase in HDL Chamomile levels; decrease in serum LDL and TGL levels; decrease in total levels of testosterone Improvement in ovarian structure and quality by reversing the deterioration of ovarian function; provides an effective compound against hyperandrogenic states; restoration of anomalies in glucose and glycosylated hemoglobin levels

Green tea (polyphenols)

Mediterranean ketogenic diet
Inhibition in testosterone secretion; decrease in TNF $\alpha$, IL- $1 \beta$, and IL-6

Decrease in weight, waist circumference, and body composition; decrease in glucose, insulin, and HOMA; hormonally, decrease in

\section{Disadvantages}

effects; increase in the risk of

cardiovascular venous thromboembolism; drug interaction should be taken into account

Minor side effects

No measurable effects upon circulating levels of TNF- $\alpha$

No evidence of the adequate dose is given; the efficacy of the combination of flutamide+EE+CA still needs to be evaluated

Contraindicated in pregnancy, as it blocks androgenic action in the formation of male external genitalia

Side effects, such as decreased libido, depression or anesthesia, and orthostatic hypotension

Side effects, such as nausea, vomiting, dyspnea, and adverse effects secondary to interactions with antibiotics and antihypertensives have been reported in some patients

Minor adverse effects; presence of mild vaginal bleeding, tachycardia, blurred vision, and even osteoporosis in some cases
Not mentioned

No side effects were observed in participants during the study period

No significant changes in the LH:FSH ratio; no significant change in DHEAS level

Increase in gastroesophageal reflux and gastrointestinal disorders

Little research on the subject

Not to use for prolonged periods of time; no visible effect in terms of improvement of oligomenorrhea nor infertility 


\begin{tabular}{|lll|}
\hline Treatment & Advantages & Disadvantages \\
\hline & $\begin{array}{l}\text { LH, total testosterone, FSH; increase in HDL, } \\
\text { estradiol, and progesterone levels }\end{array}$ & \\
\hline Aerobic exercise & $\begin{array}{l}\text { Stimulation in the reception of oxygen and } \\
\text { oxidation of free fatty acids; decrease in BMI, } \\
\text { improvement in body composition; } \\
\text { improvement in glucose circulation, thereby, } \\
\text { increase in aerobic metabolism }\end{array}$ & $\begin{array}{l}\text { No benefits observed as monotherapy on } \\
\text { reproductive function, hormonal level, or in } \\
\text { the improvement of the lipid profile }\end{array}$ \\
\hline Phlebotomy & $\begin{array}{l}\text { Decrease in insulin resistance and } \\
\text { hyperandrogenic status in women with PCOS; } \\
\text { less adverse effects on triglyceride } \\
\text { concentrations; lower prevalence of diabetes }\end{array}$ & Not mentioned \\
\hline $\begin{array}{l}\text { Cosmetic and topical } \\
\text { treatments }\end{array}$ & $\begin{array}{l}\text { Hirsutism; acne; alopecia; other } \\
\text { dermatological manifestations of } \\
\text { hyperandrogenism }\end{array}$ & $\begin{array}{l}\text { Must be complemented with medical } \\
\text { treatment; may be high or low cost, } \\
\text { depending on the conditions; may last for } \\
\text { life }\end{array}$ \\
\hline
\end{tabular}

As for the treatment made from Korean herbs, it was shown to be effective in reducing weight. This reduction is attributable to decreased energy intake and increased energy expenditure such as fat oxidation. This type of treatment caused a reduction of leptin messenger RNA and TNF expression in adipose-white tissues, Ephedra herb achieved the decrease of visceral adiposity and insulin resistance in women with obesity. ${ }^{36}$ On the other hand, it was demonstrated that the homeopathic treatment presented promising results in the management of PCOS and an improvement in quality of life, therefore, it did not alleviate only the disease itself, but the patient as a whole, but more comparative studies with a standard treatment on an adequate sample size are needed. ${ }^{37}$

Continuing with the alternatives to pharmacological treatments, Paoli et al observed that the change of diet to a eucaloric Mediterranean ketogenic diet achieved the expected results, obtaining a decrease in important components for the pathogenesis of PCOS such as weight, waist circumference, glucose, HOMA, LH, testosterone in its different forms and an increase in HDL, estradiol and progesterone. This method of treatment did not offer benefits at the level of infrequent menstruation experienced by patients or in terms of fertility, which made sense, since the change in diet had an effect mainly at the metabolic level and to achieve an improvement in the latter sections, pharmacological methods were chosen. ${ }^{32}$ In the case of aerobic exercise, this only provided certain benefits, compared to dietary change such as a decrease in BMI, better circulation of glucose and an increase in aerobic metabolism, however, it did not show improvement in other hormonal sections, therefore, it was more recommendable a change in diet, which can be complemented with exercise, to achieve a better aerobic metabolism. ${ }^{8}$

Even though suppression of androgen secretion and action with drugs that have proved great improvement rates on hirsutism can be used as spironolactone or finasteride, cosmetic or topical treatments should be considered and encouraged. ${ }^{34,35}$ Hair bleaching, chemical hair removal, shaving, among others were topical alternatives for women with PCOS that suffered from hirsutism and/or other dermatological manifestations caused by hyperandrogenism. For acne and alopecia, hair transplant surgery may be necessary in some cases as well, with the support of a dermatologist. ${ }^{34}$

The advantages and disadvantages of the above treatments are described in Table 1.

\section{Limitation}

This article had some limitations, since in some articles the population sample or the time in which different treatments were tested was small, in addition to the fact that the ethnic group varied according to the country in which they were implemented, so it was not possible to know if this type of therapy worked in the same way as in another ethnic group.

\section{CONCLUSION}

It is of vital importance to make a greater diffusion of this pathology to the general population, since it occurs in greater numbers than we think and the signs and symptoms often go unnoticed and are treated as if they were another pathology, so the treatment is often inappropriate, and the results are not as expected in patients. The fact of making an accurate and early diagnosis allows us to identify the most appropriate treatment for each case and if necessary, individualize it, according to the needs of each patient and the goals they seek to achieve as well as implement other therapeutic alternatives to optimize the results. The objective of this article was fulfilled, since it was possible to make a description of the different types of treatments that exist for PCOS, specifying the benefits that are obtained by choosing one instead of another, in addition to mentioning to which public a therapy is being directed compared to another, depending on the clinic and the characteristics presented by the patients. 
Funding: No funding sources

Conflict of interest: None declared

Ethical approval: Not required

\section{REFERENCES}

1. Borzoei A, Rafraf M, Niromanesh S, Farzadi L, Narimani F, Doostan F. Effects of cinnamon supplementation on antioxidant status and serum lipids in women with polycystic ovary syndrome. $\mathbf{J}$ Tradition Complement Med. 2018;8(1):128-33.

2. Ibáñez L, Díaz M, García-Beltrán C, Malpique R, Garde E, López-Bermejo A, et al. Toward a treatment normalizing ovulation rate in adolescent girls with polycystic ovary syndrome. J Endocrine Soc. 2020;4(5):1-9.

3. Hoffman B, Schorge J, Halvorson L, Hamid C, Corton M, Schaffer J. Williams-Ginecología. 4th ed. McGraw Hill; 2020.

4. Pani A, Gironi I, DiVieste G, Mion E, Bertuzzi F, Pintaudi B. From prediabetes to type 2 diabetes mellitus in women with polycystic ovary syndrome: lifestyle and pharmacological management. Int J Endocrinol. 2020;2020:1-10.

5. Jameson J, Fauci A, Kasper D, Hauser S, Longo D, Loscalzo J. Harrison-Principios de Medicina Interna. 20th ed. McGraw Hill; 2018.

6. Abdel-Maboud M, Menshawy A, Hasabo E, Abdelraoof M, Alshandidy M, Eid M, et al. The comparative effectiveness of 55 interventions in obese patients with polycystic ovary syndrome: A network meta-analysis of 101 randomized trials. Plos One. 2021;16(7):1-31.

7. Fraser G, Obermayer-Pietsch B, Laven J, Griesinger G, Pintiaux A, Timmerman D, et al. Randomized controlled trial of neurokinin 3 receptor antagonist fezolinetant for treatment of polycystic ovary syndrome. J Clin Endocrinol Metabol. 2021;106(9):3519-32.

8. dos Santos I, Ashe M, Cobucci R, Soares G, Maranhão T, Dantas P. The effect of exercise as an intervention for women with polycystic ovary syndrome. Medicine. 2020;99(16):1-11.

9. Pundir J, Psaroudakis D, Savnur P, Bhide P, Sabatini $\mathrm{L}$, Teede $\mathrm{H}$, et al. Inositol treatment of anovulation in women with polycystic ovary syndrome: a metaanalysis of randomised trials. Int J Obstetr Gynaecol. 2017;125(3):299-308.

10. Vanhauwaert P. Síndrome de ovario poliquístico e infertilidad. Revista Médica Clínica Las Condes. 2021;32(2):166-72.

11. Kite C, Lahart I, Afzal I, Broom D, Randeva H, Kyrou I, et al. Exercise, or exercise and diet for the management of polycystic ovary syndrome: a systematic review and meta-analysis. Systemat Rev. 2019;8(1):1-28.

12. Akbarzadeh M, Heidary M, Yazdanpanahi Z, Dabbaghmanesh M, Parsanezhad M, Emamghoreishi M. Effect of chamomile capsule on lipid- and hormonal-related parameters among women of reproductive age with polycystic ovary syndrome. $\mathbf{J}$ Res Med Sci. 2018;23(1):1-7.

13. Brenjian S, Moini A, Yamini N, Kashani L, Faridmojtahedi M, Bahramrezaie M, et al. Resveratrol treatment in patients with polycystic ovary syndrome decreased pro-inflammatory and endoplasmic reticulum stress markers. Am J Reproduct Immunol. 2019;83(1):1-10.

14. Corrie L, Gulati M, Vishwas S, Kapoor B, Singh S, Awasthi A, et al. Combination therapy of curcumin and fecal microbiota transplant: potential treatment of polycystic ovarian syndrome. Med Hypotheses. 2021;154:1-5.

15. Jazani AM, Azgomi HND, Azgomi A, Azgomi R. A comprehensive review of clinical studies with herbal medicine on polycystic ovary syndrome (PCOS). DARU J Pharmaceut Sci. 2019;27(2):863-77.

16. Mihanfar A, Nouri M, Roshangar L, Khadem-Ansari M. Polyphenols: natural compounds with promising potential in treating polycystic ovary syndrome. Reproduct Biol. 2021;21(2):1-11.

17. Meng J, Zhu Y. Efficacy of simvastatin plus metformin for polycystic ovary syndrome: a metaanalysis of randomized controlled trials. Eur J Obstetr Gynecol Reproduct Biol. 2021;257:19-24.

18. Heidari B, Lerman A, Lalia A, Lerman L, Chang A. Effect of metformin on microvascular endothelial function in polycystic ovary syndrome. Mayo Clinic Proceeding. 2019;94(12):2455-66.

19. Rezk M, Shaheen A, El-Nasr IS. Clomiphene citrate combined with metformin versus letrozole for induction of ovulation in clomiphene-resistant polycystic ovary syndrome: a randomized clinical trial. Gynecolog Endocrinol. 2017;34(4):298-300.

20. Kostopoulou E, Anagnostis P, Bosdou J, Spiliotis B, Goulis D. Polycystic ovary syndrome in adolescents: pitfalls in diagnosis and management. Curr Obes Rep. 2020;9(3):193-203.

21. Woo J, Long J. Diagnóstico Clínico y Tratamiento 2021. 60th ed. McGraw Hill; 2021.

22. Creanga A, Bradley H, McCormick C, Witkop CT. Use of metformin in polycystic ovary syndrome. Obstetr Gynecol. 2008;111(4):959-68.

23. Løvvik T, Carlsen S, Salvesen $\varnothing$, Steffensen B, Bixo $\mathrm{M}$, Gómez-Real F, et al. Use of metformin to treat pregnant women with polycystic ovary syndrome (PregMet2): a randomised, double-blind, placebocontrolled trial. Lancet Diabet Endocrinol. 2019;7(4):256-66.

24. Debras E, Fernandez H, Neveu M, Deffieux X, Capmas P. Ovarian drilling in polycystic ovary syndrome: long term pregnancy rate. Eur J Obstetr Gynecol Reproduct Biol. 2019;4:1-7.

25. Con exitoso tratamiento para ovario poliquístico en el IMSS, derechohabiente logró ser madre. IMSSUnidad de Comunicación Social. 2019;(324):1-2.

26. Ünal D, Demirci H, Yılmaz M, Kısa Ü, Tulmaç M, Güliter S. The effects of metformin, ethinyl estradiol/cyproterone acetate, and metformin ethinyl estradiol/cyproterone acetate combination therapy on 
carotid artery intima-media thickness in patients with polycystic ovary syndrome. Istanbul Med J. 2020;21(1):47-52.

27. Behboudi-Gandevani S, Abtahi H, Saadat N, Tohidi M, Ramezani Tehrani F. Effect of phlebotomy versus oral contraceptives containing cyproterone acetate on the clinical and biochemical parameters in women with polycystic ovary syndrome: a randomized controlled trial. J Ovarian Res. 2019;12(1):1-9.

28. Ghanei N, Rezaei N, Amiri G, Zayeri F, Makki G, Nasseri E. The probiotic supplementation reduced inflammation in polycystic ovary syndrome: A randomized, double-blind, placebo-controlled trial. J Function Food. 2018;42:306-11.

29. Paoli A, Mancin L, Giacona M, Bianco A, Caprio M. Effects of a ketogenic diet in overweight women with polycystic ovary syndrome. J Translation Med. 2020;18(1):1-11.

30. Muñoz M. Síndrome de Ovario Poliquístico (SOP). Revista de Formación Continuada de la Sociedad Española de Medicina de la Adolescencia. 2016;4(2):20-31.

31. Azziz R. Polycystic ovary syndrome. Obstetr Gynecol. 2018;132(2):321-36.

32. Sharma A, Welt C. Practical approach to hyperandrogenism in women. Med Clin North Am. 2021;105(6):1099-116.
33. Lee J, Jo J. Successful treatment with Korean herbal medicine and lifestyle management in an obese woman with polycystic ovarian syndrome. Integrat Med Res. 2017;6(3):325-8.

34. Oberai P, Lamba C, Manchanda R, Rath P, Bindu H, Padmanabhan M. Evaluation of homoeopathic treatment in polycystic ovary syndrome: a singleblind, randomised, placebo-controlled pilot study. Indian J Res Homoeo. 2018;12(1):35-45.

35. Al-Bayyari N, Al-Domi H, Zayed F, Hailat R, Eaton A. Androgens and hirsutism score of overweight women with polycystic ovary syndrome improved after vitamin D treatment: a randomized placebo controlled clinical trial. Clin Nutrit. 2021;40(3):8708.

36. George M, Joseph L, Chacko B, Reji N. A prospective study on the effectiveness of metformin and ethinyl estradiol-cyproterone acetate in the management of hyperandrogenism in polycystic ovary syndrome patients. World J Pharmaceut Res. 2018;7(16):116581.

37. Zhang J, Bao Y, Zhou X, Zheng L. Polycystic ovary syndrome and mitochondrial dysfunction. Reproduct Biol Endocrinol. 2019;17(1):1-15.

Cite this article as: Llamas MC, Montejano JM, Vergara MDCM, Muñoz AZC, Vargas MGR, Otero AL, et al. Comparison of different treatments used for polycystic ovary syndrome. Int J Reprod Contracept Obstet Gynecol 2022;11:644-54. 